\title{
Plasma whole-genome sequencing put to the test
}

New research published in Genome Medicine has assessed plasma genome sequencing in men with castrationresistance prostate cancer (CRPC) and hormone-sensitive prostate cancer, finding that, not only is plasma wholegenome sequencing viable, quick and cheap, it also seems to be accurate.

\section{4 ...high copy number gains} in the androgen receptor locus were observed in patients with CRPC... 77

Little is known about the late genetic alterations acquired in the tumours of men with CRPC because the lesions tend to metastasize to bone, which is challenging to access for biopsy. Consequently, 'liquid biopsy' techniques-which use blood samples to assess the genetic landscape of patients with cancer-have garnered much attention.
"We explored whole-genome sequencing of plasma DNA using existing sequencing-by-synthesis chemistry technology," explains lead investigator Michael Speicher. The researchers analysed samples from 25 individuals without cancer and nine patients, five of whom had CRPC. They revealed multiple copy number aberrations in the patients with cancerincluding losses in $8 \mathrm{p}$ and gains in $8 \mathrm{q}$, which have been previously identified in prostate tumours.

Unsurprisingly, high copy number gains in the androgen receptor locus were observed in patients with CRPC, but not in those with hormone-responsive disease. "Additionally, the TMPRSS2:ERG rearrangement occurs in $50 \%$ of all prostate cancers and is associated with a 3-Mbp deletion on chromosome 21," continues Speicher. "With our approach, we were able to identify even such a small deletion and the corresponding fusion plasma fragments in these cases."
Perhaps illustrating the potential clinical utility of the method, the researchers identified chromosomal rearrangements in one patient that had not been revealed in the previous analyses of his tumours. These rearrangements were consistently identified in serial plasma analyses over a 9-month period, which is consistent with the presence of one metastatic clone.

Arguably, identifying any actionable mutations as early as possible in the disease history will facilitate personalized treatment, and this high-throughput technique seems to offer this opportunity. Although at an early stage, this research is a step in the right direction towards realizing reliable liquid biopsy methods. Larger clinical studies, encompassing more patients, are planned.

Mina Razzak

Original article Heitzer, E. et al. Tumour associated copy number changes in the circulation of patients with prostate cancer identified through whole-genome sequencing. Genome Med. doi:10.1186/gm434 